Pecvnia, 5 (2007), pp. 17-27

\title{
¿Qué realidad representa la contabilidad?
}

\author{
Walter Carrizo \\ wcarrizo@unpata.edu.ar \\ Silvia León \\ silvialeon_unp@yahoo.com.ar \\ Universidad Nacional de La Patagonia San Juan Bosco \\ Fac. de Ciencias Económicas Comodoro Rivadavia \\ Ciudad Universitaria $3^{\circ}$ piso - Ruta Provincial $n^{\circ} 1 \mathrm{~s} / \mathrm{n}$ \\ 9000 Comodoro Rivadavia - Provincia de Chubut (Argentina)
}

La discusión acerca del status epistemológico de la contabilidad sigue aún vigente. A pesar de ello, los autores coinciden en general, en que el problema central de esta disciplina radicaría en producir información acerca de la realidad en la que se encuentra un determinado ente económico en cuestión. Para ello se recurre a segmentar la realidad, modelizarla y medirla, de modo de poder representarla y así ejercer control sobre tal ente. Es aquí donde surge el siguiente interrogante ¿cuál es la realidad que representa la contabilidad?

Para responder a esta pregunta es necesario analizar las distintas concepciones acerca del término "realidad" según las distintas corrientes y aportes de pensadores. Así, de una concepción de realidad meramente observable
The discussion about the epistemological status of accounting is still a vivid issue. Nevertheless, there is a general consensus among the most important authors, that the core problem of this discipline might lie in the ability to produce information about the reality in which an economic entity develops its actions. In order to fullfill this goal, reality is segmented, modernized and measured, so that it can be represented and the control over the entity achieved. Considering this theoretical frame, a question came to our minds: what reality does accounting represent?

In order to properly answer this question, the different ideas about the term "reality" proposed by different schools and authors should be analized. So, from an idea of a merely observable reality, which can only be described, 
para su descripción, podemos pasar a otra en la que la observación no es un simple acto pasivo sino que asume un cariz práctico - operativo. Los conocimientos generados accionarán sobre la realidad y sus implicancias futuras.

Entendida como una tecnología social, el propósito de la contabilidad, más que representar la realidad para brindar una explicación o descripción de la misma, consiste en ser un instrumento efectivo al momento de actuar generando cambios en el ente objeto de estudio.

Palabras clave: Contabilidad. Realidad, representación, modelo, medición, tecnología social, instrumento efectivo. another idea can be considered in which the observation is not just a passive action, but an action that can assume a practical and operational profile. The generated knowledge will influence the reality and its future implications.

Assuming accounting as a social technology, the main goal of our discipline, more than representing reality only to offer an explanation or a description of it, would consist in being an effective tool when the generation of changes is required.

Key words: Accountancy, reality, representation, model, measurement, social technology, effective tool.

\section{INTRODUCCIÓN}

El presente trabajo surge como un subproducto del proyecto de investigación "La teoría de la medición en la contabilidad - Su estado actual en la Argentina".

A raíz de la revisión de la bibliografía existente en nuestro país, el concepto de medición está presente en toda la producción literaria especializada, sea en la definición de la disciplina o reconocida como una de sus tareas sustantivas.

Asumiendo que la acción de medir intenta transferir aspectos cualitativos de la realidad a una apreciación en forma cuantitativa, de modo de lograr una representación de la misma, nos surge la siguiente reflexión: ¿cuál es la realidad que representa la contabilidad? Tal acción de representar, iimplica solamente una actividad descriptiva o es la contabilidad un agente activo que puede influir en la realidad representada? ¿La representación es estática o puede generar cambios en la realidad futura de un ente?

Sin la pretensión de que éste sea un estudio acabado de esta problemática, nos proponemos revisar algunos conceptos y posturas acerca del término "realidad", que nos permitan dilucidar un esquema acorde al encuadre de la disciplina contable desde la postura de una tecnología social. 


\section{UN ENCUADRE EPISTEMOLÓGICO DE LA CONTABILIDAD}

El estudio de la contabilidad como rama del conocimiento, nos lleva a preguntarnos acerca de su status epistemológico, de modo de establecer su objeto de análisis, su finalidad y los medios que posee para abordar su problemática y como la resuelve. Variada es la visión de los principales autores de nuestro país acerca de este tema. Podemos resumir tres posturas diferentes:

1. una corriente de pensadores sostiene que la contabilidad es una ciencia factual, dedicada a la comprensión de la realidad, y a cuyos efectos desarrolla técnicas de medición para poder describirla.

2. otros estiman que la contabilidad es una tecnología social, abocada a reconocer, clasificar y medir un cierto segmento de la realidad.

3. hay autores que no desarrollan una concepción epistemológica de la disciplina. Sin embargo, de sus conceptos se desprende una clara visión de la contabilidad como una técnica.

Más allá del encuadre en el que se encuentren, todos los autores coinciden de alguna u otra forma en la existencia de un problema central, cual es el producir información acerca de la realidad en la que se encuentra un determinado ente económico en cuestión. Esta información debe ser útil para la toma posterior de decisiones y haber sido brindada bajo parámetros de objetividad y equidad, que permitan su comparabilidad a través del tiempo y paralelamente entre distintos entes que compartan el mismo segmento de realidad. Para ello se recurre a segmentar la realidad, modelizarla y medirla. Específicamente esta última acción de medir se convierte en el centro de atención de los desarrollos teóricos, ya que a través de ella se podrá expresar la realidad observada.

En función de esta coincidencia, nos parece especialmente útil la distinción de Dussel:

La inteligencia que integra el acto humano puede situarse en posición teórico-contemplativa o práctico operativa. En el primer caso decimos que se abre al ámbito de la verdad teórica o que da cuenta de la realidad dada, en el segundo decimos que se abre al ámbito de la verdad para la acción o que funda no la realidad dada, sino la realidad que se efectuará en el futuro y por mediación de la misma acción (1984: 189). 
No parece haber posibilidad de pensar una contabilidad que simplemente intente describir o explicar fenómenos. Siempre el fin es utilizar la información con algún propósito. Así, la información generada por el sistema contable no solo "describe" tres aspectos de la realidad de un ente (realidad patrimonial, económica y financiera), sino que generará decisiones que podrían modificar la realidad empresaria y la de su contexto. Pensemos en el siguiente caso: de los estados contables de una empresa se puede determinar la evolución de sus negocios, de su rentabilidad, de su posición en el mercado, etc. Estos aspectos podrían inducir a una persona u organización a invertir en ella, generando así cambios no solo en la realidad de la empresa, sino además en la suya propia, al afectar su patrimonio.

Si analizamos aspectos más cotidianos de esta disciplina, el sistema contable, como instrumento de captación y transformación de datos en información, consta además de mecanismos de almacenaje cuyo análisis periódico y constante permitiría detectar desvíos, abriendo así caminos de corrección. Esto permite que se produzca, según Gil (1999: 25) "información pertinente, oportuna y predictiva". Esta capacidad de predicción nos lleva a pensar también en su capacidad de modificación de la realidad futura.

De todas formas, la más simple y elemental actividad de confeccionar los estados contables que representan la realidad del ente, conlleva consecuencias futuras, de no cumplirse en los términos legales de tiempo y forma.

El viejo principio de "significatividad", presente en las actuales Resoluciones Técnicas a través del concepto de "desvíos aceptables", nos enfrenta a la posibilidad de que la representación ofrecida por la información contable se aparte en alguna medida de su objeto representado, siempre que tales desvíos no influyan erróneamente en las futuras decisiones.

A todo esto, el profesional auditor debe emitir una opinión acerca de los informes producidos por la contabilidad de un ente, en la que expresará si ellos representan, o no, razonablemente la realidad de ese ente. Esta acción influirá directamente en el comportamiento de los usuarios de la información, en sí potenciales decididores futuros.

Dados estos aspectos vertidos, se pone en cuestión una idea central: ¿existe una realidad objetiva cuya naturaleza puede ser determinada empíricamente? Si esto fuera así, tiene sentido concentrar 
todos los esfuerzos en describir y evaluar la situación de un ente, así como sus resultados, de modo de proveer a los decididores de fundamentos para sus decisiones.

Pero, si avanzamos en la línea argumentativa en la que nos hemos situados, esa realidad es siempre realidad producida a partir de acciones intencionales tomadas sobre la base de la información proveída. Nuevamente, dice Dussel:

La verdad teórica concuerda con lo real a priori; la verdad para la acción hace concordar lo real con lo proyectado a posteriori (1984: 189).

Por otro lado, ¿cómo se establece el criterio de razonabilidad del que debe estar imbuida la representación contable de la realidad empresaria? Este criterio, ¿segmentaría aún más la realidad observada?

Para tratar de dilucidar algunas respuestas a estas preguntar, sería menester analizar los encuadres filosóficos que nos ayuden a determinar a qué nos referimos cuando hablamos de "realidad".

\section{LAS POSTURAS FILOSÓFICAS ACERCA DE LA REALIDAD}

Término tan cercano a nuestra vida cotidiana, la realidad se relaciona directamente con el concepto de lo real. Etimológicamente esta palabra proviene del latín y quiere decir literalmente "cosa", en relación directa a la materia física, susceptible por lo tanto, de ser observada, estudiada, medida, controlada y transformada. ¿Cómo realizar esta observación? ¿El observador es ajeno a ella o puede influirla? Es en este dilema donde aparecen distintas posturas.

En primera instancia, podemos hablar de una corriente "realista". Según esta postura, existe un mundo real y objetivo, independiente de los seres humanos, con una naturaleza o entidad propia, susceptible de ser cognoscible. La esencia natural de este mundo se considera única e inmutable, y las acciones que el observador realice para estudiarla no la modifican. Por tal motivo, ésta puede ser descrita y corroborada empíricamente. Esta línea de pensamiento se encuentra vastamente representada en la investigación contable actual.

Otra postura está dada por aquellos que piensan que la realidad es creada y recreada en una constante relación entre los teóricos y la realidad misma. Así la realidad social se construye gracias a la 
intervención del lenguaje y la interpretación de lo medido o descrito. En esta línea de pensamiento se encuentra la escuela interpretativa inglesa.

Otro aporte acerca de la idea de realidad sugiere que la misma está constituida por diferentes niveles o capas en lo que se ha dado en llamar según Mattesich (2006: 6) el "onion model of reality" o modelo de la realidad en capas de cebolla. Según éste, la realidad está compuesta por distintos niveles o capas "dependientes de e incluidas una en la otra". Se conciben estos niveles no ya desde una perspectiva lineal sino sobre todo, desde la idea multidimensional que incluya el tiempo y otras más. Así, estas capas no serán consideradas como estáticas sino que se verán alteradas, a excepción de la que conforma el núcleo, quizás a un nivel de energía pura. La idea de que cada elemento de la realidad posee propiedades propias que al verse expuesto a la influencia de otros, o combinados con ellos, produce "propiedades emergentes" únicas que nos obligan a pensar en qué consideramos real en cada capa. Según este modelo la realidad estaría constituida por los siguientes niveles:

1. realidad físico-química

2. realidad biológica

3. realidad mental

4. realidad social.

\section{LA IDEA DE REALIDAD EN LA CONTABILIDAD}

Sea la postura en la que nos enrolemos, se reconoce que la contabilidad trabaja sobre una realidad social, ya que gran parte de los conceptos que emplea se refieren a aspectos que se desarrollan en el ámbito de las relaciones entre humanos y los grupos sociales que ellos conforman, como por ejemplo las ideas de propiedad, control, deudas y obligaciones, entre otras.

Sin embargo, la pregunta epistemológica de ¿cuánto podemos conocer de esa realidad? dependerá de la visión que tengamos de ésta. Así, para un realista descriptivo, la contabilidad observará y captará hechos, reales en el plano de lo social, y los transformará en información útil de modo de representar la realidad de un ente, hecho que le permitirá controlarlo. 
Mattesich, por el contrario, propone un modelo de representación orientado al objetivo. De esta forma, conceptos tales como ingreso o capital, se consideran reales en el plano de la realidad social, pero profundamente ligado a aspectos metodológicos de medida o estimación de valores, temporalmente atribuidos a tales concepto, es decir, teniendo en cuenta el objetivo que se persiga con la información generada. El esquema presentado resalta la importancia central de buscar una representación orientada al objetivo. Esto quiere decir que se buscará emparejar los medios apropiados a unos fines correctamente especificados. Para ello es necesario establecer dos requisitos previos:

1. la aceptación de varios modelos, dependiendo de cada uno de un objetivo específico de la información.

2. un análisis profundo de los medios-fines.

Otras contribuciones sobre el aspecto ontológico de la realidad están dadas por Baudrillar al hablar de hiperrealismo y sobre por las aplicaciones que el grupo Macintosh ha querido hacer de esta idea a la contabilidad. Según esta postura, las representaciones de la realidad no son sino meros simulacros de ella, los que ya no se asocian con ningún referente real y donde los símbolos, las imágenes y los modelos circulan a cualquier objeto material real. El equipo Macintosh llega a la conclusión que no se puede hacer una distinción entre realidad y representación, es decir, la representación mental es la única realidad. Según Mattesich, esta postura podría llevarnos a un estado paranoico esquizofrénico. Aplicando estos conceptos a la contabilidad, centrar el accionar en la representación mental podría llevar a distorsiones deliberadas que induzcan a creer en una realidad inexistente, tal el caso de lo ocurrido con el grupo Enron y de los efectos que tuvo la información acerca de su realidad. ¿Será esta postura el fundamento de la llamada "contabilidad creativa"?. Ahora bien, si toda representación mental se hace presente en el lenguaje, estamos obligados a plantear la relación entre los enunciados de nuestro lenguaje y la representación mental de la realidad que origina estos enunciados.

Así, en todo proceso de conocimiento, la mediación del lenguaje no puede ser desconocida. Todo lo que creemos saber acerca de la realidad, lo expresamos en enunciados acerca de ella. Por consiguiente, ¿Cuándo es verdadero lo que decimos acerca del mundo? Intuitivamente, y esto es un lugar común, entendemos que un enunciado es verdadero si se corresponde con hechos de la realidad. Esta concepción, conocida 
como correspondentista, es hoy altamente cuestionada y se habla de verdad como coherencia, afirmabilidad garantizada, afirmabilidad idealmente garantizada, aquello que la ciencia terminará por sostener, aquello que explica el éxito de la ciencia o de nuestras creencias comunes, etc. $Y$ en el centro de este debate, la noción de representación no puede ser obviada.

Autores como Rorty rechazan la representación y la consideración de la verdad como correspondencia. No es posible determinar si ciertas entidades contables existen a partir de la aparición de la palabra que las nombra, o si la palabra es posterior a la existencia de la cosa, puesto que es el lenguaje quien nos da a conocer la cosa. Pensemos en conceptos claves para la disciplina contable y para el mundo de los negocios: todo ente económico necesita de un capital para funcionar y cumplir con sus objetivos. Ahora bien, definido como la inversión inicial necesaria para comenzar las actividades económicas, podemos determinar la igualdad básica de la que parten todos los razonamientos de la contabilidad patrimonial:

$$
\text { Activo }=\text { Pasivo }+ \text { Capital }
$$

Si tomamos en cuenta que al iniciar un negocio las personas involucradas en la creación de un ente económico aportan solo recursos, llegamos a la conclusión que en ese momento:

$$
\text { Activo }=\text { Capital }
$$

¿Es el ACTIVO realmente igual al CAPITAL? ¿Existe el CAPITAL como entidad tal? ¿O es la idea del aporte medido en dinero la que da nacimiento al concepto de capital y que nos sirve para representar la realidad del ente mediante un concepto de dualidad y de igualdad?

Profundizando esta línea de pensamiento, el concepto de RESULTADO se relaciona con la mayor o menor riqueza generada por un ente, teniendo en cuenta un parámetro de referencia, el CAPITAL. Un contador puede informar el resultado obtenido por un ente, pero esta cifra difícilmente será entendida por el empresario si no se aportan los aspectos financieros que éste involucra. Entonces, ¿existe en sí el resultado? ¿O es un concepto que satisface a los contadores para explicar las diferencias obtenidas por la aplicación de criterios de medición, o al realizar comparaciones temporales?

Dice Romeo César: 
El desechar la representación, las instancias extralingüisticas y por ende cualquier consideración de la verdad como correspondencia, le permite a Rorty llegar tras los pasos de William James a su famosa y controvertida formulación: "verdad es lo que es bueno para nosotros creer" (2004: 6).

Verdad para nosotros es que los bienes se incorporan a su valor de contado. Si la operación se realizó en forma financiada, los intereses no forman parte de ese costo de adquisición, sino que son el valor de otro tipo de operación. Sin embargo, para el dueño del bien, la verdad es el monto que finalmente terminó pagando. $Y$ sostiene Romeo César más adelante:

... (Rorty) considera que el calificativo "verdadero" es un cumplido hecho a aquellas creencias nuestras a las que juzgamos tan bien justificadas que, por el momento, no necesitamos para ellas de ninguna justificación ulterior (2004: 7).

En esta línea de cuestionamiento, y asociado al comienzo de nuestra exposición, "la verdad para la acción o que funda... la realidad que se efectuará en el futuro y por mediación de la misma acción", la "realidad" que la contabilidad "representa" queda definitivamente atada a los propósitos que persigue quien elabora, diseña y trasmite la información.

\section{CONCLUSIONES}

En nuestra opinión, la contabilidad, en tanto tecnología social, genera información que intenta representar una realidad sobre la que ella misma ha influido y modificará sin lugar a dudas con el sólo hecho de emitir un enunciado al respecto. Esta realidad "representada" incluye ideas, conceptos, que se articulan en un discurso asumido como verdadero a los efectos de poder explicar la situación de su objeto de estudio, el ente económico, para accionar sobre él. A ello debemos sumarle la presencia de convenciones generalmente aceptadas y que en nuestro tiempo han alcanzado fuerza coercitiva, como es el caso de las normas contables, nuestras Resoluciones Técnicas, que si bien parecieran uniformar los criterios, no es su propósito asegurar una representación "verdadera" de la realidad. En todo caso sólo pretenden unificar el discurso para algunos fines muy acotados, quedando otra infinidad de situaciones libradas al profesional que produce la información, como en el 
caso de la contabilidad gerencial o en contabilidad de costos. En este sentido, el modelo no es único sino que dependerá de los objetivos que persiga el decisor en función del cual fijará los indicadores necesarios para un análisis específico, las categorías a utilizar y la escala.

Así sólo se registran los hechos contingentes negativos, los cuales formarán parte de la "representación" de la realidad que brinda la contabilidad. Por otro lado, aquellos hechos contingentes positivos se informan, pero no en el cuerpo central de los Estados Contables, sino como una nota complementaria, realizando por lo tanto, un recorte intencional en la "realidad representada" (enmascarado tras un principio de "prudencia").

La concepción de la contabilidad como tecnología social se funda en el hecho de que su propósito, más que representar la realidad para brindar una explicación o descripción de la misma, consiste en ser un instrumento efectivo al momento de actuar generando cambios en el ente objeto de estudio. En palabras de María Cristina Wirth:

la medición contable del rendimiento económico no es una representación pasiva del mundo de los fenómenos reales, sino un agente activo que afecta el mundo real al influenciar al decididor (2001: 39).

Cambiando finalmente el punto de vista en el abordaje de la cuestión, ¿cómo pensar que nuestra función, en tanto docentes, es mediar en el proceso de construcción del conocimiento contable, si no es desde una posición como la planteada?

\section{BIBLIOGRAFÍA}

Centro de Estudios Científicos y TÉCnicos (CECyT) (2004) Resoluciones Técnicas. Buenos Aires: Federación Argentina de Consejos Profesionales en Ciencias Económicas (FACPCE).

CÉSAR, Romeo (2004) Sobre la verdad. Separata 2. DAVIDSON, RORTY, APEL. Facultad de Humanidades y Ciencias Sociales, Universidad Nacional de la Patagonia San Juan Bosco.

DAVIDSON, D. (1987) "Epistemología y Verdad", Actas Congreso Internacional de Filosofía, Tomo I, pp. 148-179. 
DUSSEL, Enrique (1984) Filosofía de la producción. Bogotá: Editorial Nueva América.

García Cassella, Carlos; Ramírez de Rodríguez, María del Carmen (2001) Elementos para una Teoría General de la Contabilidad. Buenos Aires: Editorial La Ley.

GIL, Jorge Manuel (1999) "El derecho contable y los estilos de administración en el marco de la globalización económica: apuntes para una revisión conceptual y situación actual en América Latina", Actas de las V Jornadas de Epistemología de las Ciencias Económicas.

MATTESICH, Richard (2001) Contabilidad y Método Analítico. Medición y Proyección del Ingreso la riqueza en la Microeconomía y en la Macroeconomía. Buenos Aires: Editorial La Ley.

- (2006) La Representación Contable y el Modelo de Capas - Cebolla de la Realidad: Una comparación con las "Ordenes de Simulacro" de Baudrillard y su Hiperrealidad. Buenos Aires: Centro de Investigación en Epistemología de las Ciencias Económicas, Facultad de Ciencias Económicas, Universidad de Buenos Aires.

MORETTI, A. (1996) Concepciones tarskianas de la verdad. Buenos Aires: Oficina de Publicaciones del Ciclo Básico Común, Facultad de Filosofía y Letras, Universidad de Buenos Aires.

SCARANO, Eduardo (1995) "La contabilidad y la aparición de la tecnología ¿un caso de involución?", Actas Primeras Jornadas de Epistemología de las Ciencias Económicas, pp. 139-148.

WIRTH, María Cristina (2001) Acerca de la ubicación de la Contabilidad en el Campo del Conocimiento. Buenos Aires: Editorial La Ley. 